\title{
Gestão Pública e Pesquisa O peracional: avaliação de desempenho em Agências da Previdência Social
}

A delina Cristina A . Chaves e A . Clecio F. Thomaz

\section{Introdução}

A Era da Informação, apoiada em Ciência e Tecnologia, vem produzindo uma extraordinária mutação no sentido de expandir a consciência da sociedade quanto às suas expectativas e necessidades. A qualidade dos produtos e serviços deixa de ser uma alternativa organizacional para assumir o papel de demanda social. Estudiosos afirmam que a competitividade e a sobrevivência de uma organização dependerão de sua agilidade, flexibilidade e capacidade de inovação. Mas, segundo Goldbarg e Luna (2000), "sem buscarmos o ideal da otimização não poderemos criar uma plataforma firme onde a criatividade e a visão empreendedora possam se sustentar".

O Estado, por sua vez, não pode ficar indiferente à evolução de seus usuários. Assim, a busca de qualidade e produtividade chega à G estão Pública como um mecanismo de fortalecimento institucional. Contudo, "a gestão pública carece de instrumentos para implementação de medidas integradas em agendas de 
transformações da gestão que assegurem eficiência e efetividade à ação do Estado na consecução dos serviços públicos" (BRASIL, 2003).

A Previdência Social, segundo França (2004), "reduz as desigualdades sociais e exerce uma influência extraordinária na economia de um incontável número de municípios brasileiros". França apresenta em seu livro "A Previdência e a Economia dos Municípios" números "reveladores da magnitude de uma estrutura [...] que precisa ser cada vez mais aperfeiçoada, em benefício de toda a sociedade brasileira".

Este artigo trata de avaliação da eficiência de Agências da Previdência Social (INSS) utilizando a metodologia
DEA (D ata E nvelopment A nalysis). Para proceder à avaliação, foi implementado um modelo DEA-CCR utilizando o software DEA-Solver@ (CoOPER, Seiford, Tone, 2000). As informações foram obtidas do Suibe - Sistema Único de Informações de Benefícios da Previdência Social, sendo analisado o período de novembro de 2006, que foi o mês de referência para implantação da reestruturação da carreira do servidor. 0 estudo se desenvolveu na Gerência Executiva Fortaleza que, para 0 atendimento direto aos beneficiários, conta com 22 Agências da Previdência Social (APS), sendo 10 na região metropolitana de Fortaleza e 12 em outros municípios cearenses de sua área de abrangência (figura 1).

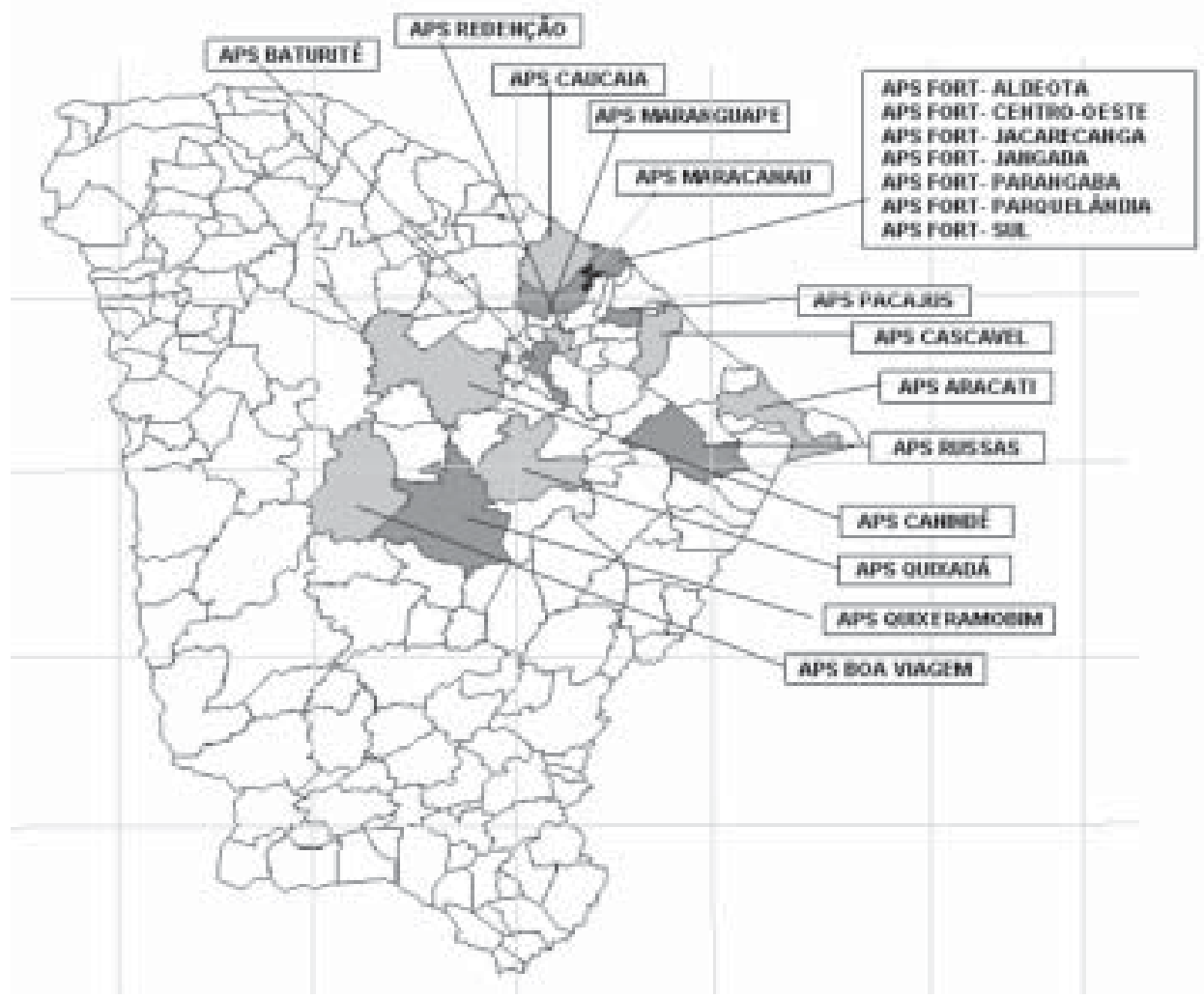

Figura 1: Distribuição Espacial das APS-GEXFOR - Mapa do Ceará 


\section{Análise de eficiência relativa}

A Análise Envoltória de Dados DEA (D ata E nvelopment A nalisys) - possibilita avaliar o grau de eficiência relativa de unidades produtivas as quais realizam uma mesma atividade, quanto à utilização dos seus recursos. A análise da eficiência de unidades produtivas, D MU (D edsion M aking U nit), nos modelos DEA, gera uma fronteira de eficiência sobre a qual estarão situadas as unidades eficientes, ou seja, as que possuírem a melhor relação "produto/ insumo". As unidades menos eficientes estarão situadas numa região inferior à fronteira, conhecida como envoltória. Os modelos DEA transformam os inputs (insumos) e outputs (produtos) originais pela agregação de valores, em combinação linear de inputs e outputs, respectivamente. O s pesos usados nestas combinações lineares são calculados por um problema de programação linear, de forma que cada D MU obtenha a melhor combinação de pesos, maximizando sua eficiência.

Em qualquer modelo DEA:

1) Para cada DMU, escolhe-se seu respectivo conjunto de pesos, de modo que cada DMU apareça o melhor possível em relação às demais. D essa forma, cada DMU pode ter um conjunto de pesos (multiplicadores) diferente;

2) Todos os modelos são invariantes com a escala de medida;

3) A DMU que apresentar a melhor relação (output j) / (input i ) será sempre eficiente;

4) Há pré-escolha das variáveis, ou seja, identificação de quais variáveis poderão compor o modelo. Poderão ser levados em conta fatores controláveis (de gestão) e fatores não controláveis (do ambiente), tanto qualitativos, como quantitativos.
5) As D MU escolhidas precisam estar alinhadas e desempenhando funções semelhantes.

A metodologia DEA apresenta ainda as seguintes vantagens:

- Os dados não necessitam de normalização;

- É uma abordagem não paramétrica, não exigindo uma forma funcional explícita relacionando input e output;

$\quad$ “O Estado não
pode ficarindiferente
à evolução de seus
usuánios. Assim, a
busca de qualidade
e produtividade chega
à Gestão Pública como
um mecanismo de
fortalecimento
institucional."

- O s índices de eficiência são baseados em dados reais (e não em fórmulas teóricas);

- Generaliza o método de Farrell, construindo um único input virtual e um único output virtual;

- Pode, explicitamente, sinalizar a não eficiência do processo de produção e também apontar possíveis melhoramentos relacionados aos investimentos; 
- Ao contrário das abordagens paramétricas tradicionais, a DEA otimiza cada observação individual com o objetivo de determinar uma fronteira linear por partes (piece-wise linear) (Figura 2), que compreende o conjunto de D MU ParetoEficiente.

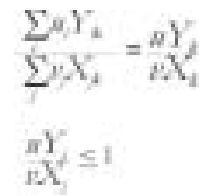

Onde:

$\mathrm{k}=1, \ldots, \mathrm{n}$ DMU

$\mathrm{i}=1, \ldots, \mathrm{m}$ inputs de cada DMU

$\mathrm{j}=1, \ldots, \mathrm{s}$ output de cada DMU

outputs (produtos) e inputs (insumos). 0 método convenciona que todos os

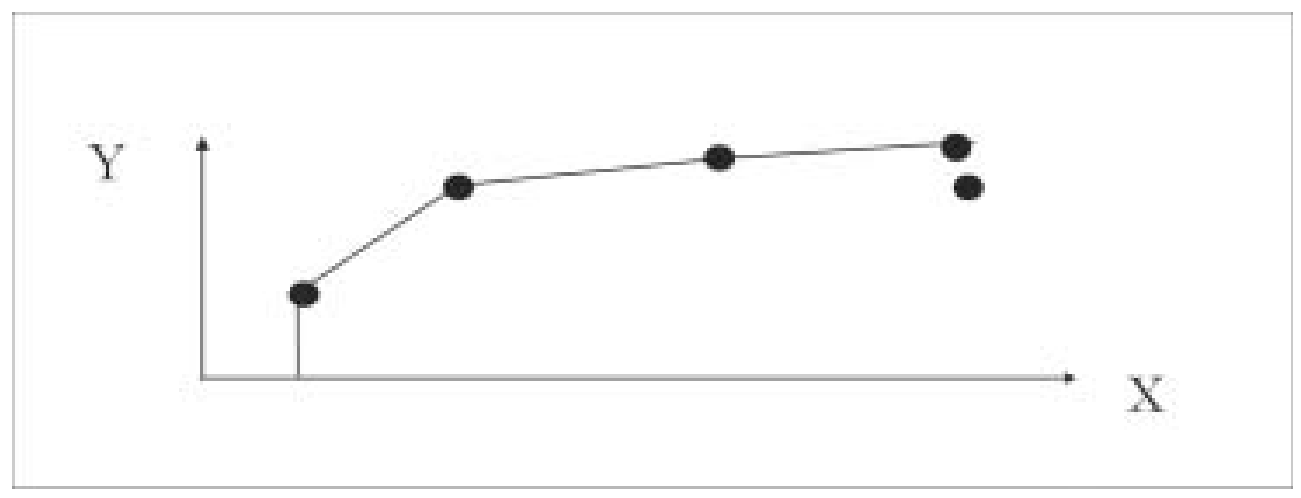

Figura 2: Fronteira linear por partes (piece-wise linear)

Essas características conferem ao método uma potencialidade de natureza essencialmenteaplicada. DEA éum método para apoio à decisão de natureza multicritério e, portanto, capaz de modelar melhor a complexidade do mundo real.

$\mathrm{O}$ modelo CCR (Charnes, Cooper e Rhodes), também conhecido como CRS (Constant Returns to Scale) tem como propriedade principal a proporcionalidade entre inputs e outputs na fronteira, ou seja, 0 aumento (decremento) na quantidade dos inputs provocará acréscimo (redução) proporcional no valor dos outputs. Considerando inputs X I (input X de cada unidade i) e os outputs $Y j$ (output $Y$ de cada unidade j), 0 índice de eficiência é definido pela combinação linear dos outputs dividido pela combinação linear dos inputs de determinada DMU $\mathrm{k}$ :

Nesta expressão, u e v representam os pesos ou multiplicadores atribuídos aos índices devem ser menores ou iguais a 1. O u seja, quanto maior a relação Y/X maior a eficiência da unidade. A eficiência de uma D MU A será calculada por meio do problema de programação não-linear seguinte:

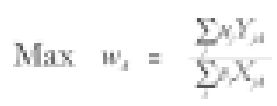

tal que

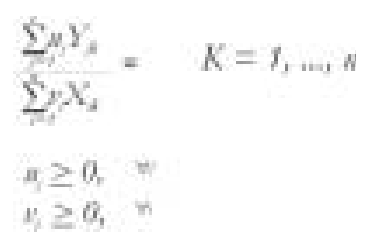

Então o problema consiste em encontrar os valores dos pesos (ou multiplicadores) ui e vj, que maximizam a soma 
ponderada dos outputs (output "virtuais") dividida pela soma ponderada dos inputs ("inputs virtual") da DMU em análise, sujeita à restrição de que este quociente seja menor ou igual a 1, para todas as DMU. Logo, 0 índice de eficiência varia de 0 a 1, sendo considerada eficiente a unidade que obtiver índice igual a 1, e as demais, ineficientes.

Repetindo-se esse processo para cada D MU obtêm-se os respectivos pesos ui e vj.

Charnes e Cooper (1962) transformaram o Problema de Programação Fracionária acima, que tem infinitas eótimas soluções, num Problema de Programação Linear (PPL):

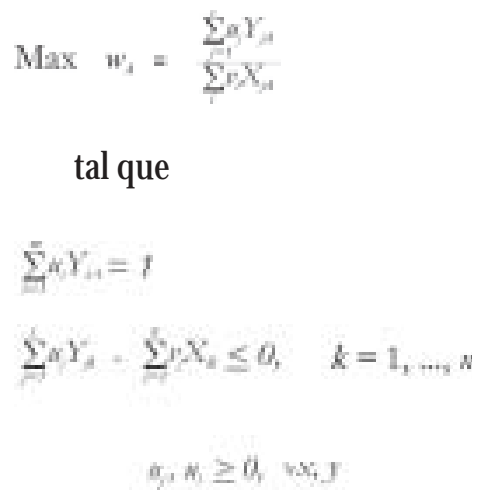

Este modelo assume retornos constantes de escala (CRS), a partir dos pesos atribuídos aos inputs (insumos) e outputs (produtos).

\section{E studo de Caso: Análise de Eficiência das APS-GEXFOR}

Uma Agência da Previdência Social é basicamente uma entidade prestadora de serviços à comunidade, sendo a instância organizacional que lida diretamente com seus usuários. Além dos sistemas corporativos, a instituição conta com sistemas de informação como: o Sistema Único de Informações de Benefícios - que integra e concentra, num sistema único, informações gerenciais, originalmente dispersas em várias fontes, permitindo integração e visão global do negócio cidadão-segurado; 0 SGA - Sistema de G estão do A tendimento, que permite o gerenciamento do atendimento aos beneficiários nas unidades; e o SAE - Sistema de Agendamento Eletrônico, o mais recente instrumento da Previdência Social para melhoria do atendimento, que permite aos usuários agendar eletronicamente seu atendimento, escolhendo data, hora e unidade que lhes seja mais conveniente, sendo acessado tanto pela internet quanto através da central de atendimento, pelo telefone gratuito 135.

\section{Insumos e produtos}

Os dados relativos aos processos de benefícios foram obtidos por meio do Suibe, e os dados relativos ao quantitativo de servidores nas unidades alcançados a partir dos sistemas da Diretoria de Recursos Humanos. Inicialmente, foram consideradas as seguintes variáveis: Requer - variável não controlável, que representa 0 quantitativo de requerimentos aguardando despacho conclusivo da APS; $\mathrm{N}{ }^{\circ}$ Servidores - variável controlável, que demonstra o quantitativo de servidores lotados na APS; D espach - constitui o quantitativo de requerimentos que tiveram despacho conclusivo na unidade: (deferimentos e indeferimentos).

As demais variáveis foram definidas a partir de indicadores institucionais, sendo considerados (preferencialmente) aqueles com ampla divulgação nas diversas instâncias gerenciais da instituição e que melhor representam as atividades-fim. São elas: ICTU - representa o percentual de benefícios despachados na unidade, considerando o grau de complexidade dos mesmos, e de acordo com um padrão 
mínimo estabelecido pela instituição; IPD U - representa ao percentual de benefícios despachados por servidor; ICA - representa a quantidade média de requerimentos efetuados por servidor; ID T - representa 0 índice de demanda atendida; D TC representa a distribuição do tempo de concessão; D T R - representa a distribuição do tempo de represamento; IM A - representa a idade média do acervo (no modelo usamos a variável IM A r - o indicador invertido); e TMC - representa o tempo médio concessório (no modelo usamos a variável $\mathrm{TMCr}-0$ indicador invertido).

As variáveis inicialmente listadas foram submetidas aos processos recomendados pelos autores da literatura consultada e submetidas ao método de Norman e Stoker (1991), que alia a Análise de Correlação Simples à Análise Envoltória de D ados para definir aquelas que comporiam o modelo a ser implementado, definindo quais seriam insumos e quais seriam produtos. Para desenvolvimento da aplicação, foi utilizado o modelo "DEA CCR-O.CCR" orientado a output, que tem como objetivo maximizar os produtos obtidos com, no máximo, o mesmo nível dos inputs. 0 modelo foi implementado pelo sotware DEA-Solverø.

\section{Seleção das variáveis para aplicação do modelo DEA}

As variáveis pré-selecionadas foram submetidas a procedimentos baseados no método de NORMAN e STOKER (apud Paiva, 2000; SILVA, 2000) para que se definisse sua inclusão ou não no modelo. A partir desse procedimento é construída uma seqüência de indicadores de eficiência para os planos de operação observados. Inicia-se com a classificação das variáveis em input (insumo) e output (produto) de acordo com o desempenho observado.
Para iniciar o método, seleciona-se inicialmente um par: input-output e aplica-se o modelo DEA-CCR, obtendo-se, assim, as medidas de ineficiência dos planos de operação observados.

Seqüencialmente, novas variáveis são incluídas, tomando como base o grau de correlação entre a medida DEA obtida e os insumos e produtos considerados na análise de eficiência. Levando-se em conta que a correlação dos produtos com o indicador de eficiência deveria ser positiva, um aumento no volume de produtos acarreta um aumento no indicador de eficiência.

O raciocínio oposto deve ser aplicado aos insumos, implicando correlação negativa com o indicador eficiência. Portanto, se a correlação calculada entre um indicador de eficiência e uma variável de output (produto) for negativa, os efeitos desta variável não estão computados no indicador calculado. D etermina-se a necessidade de inclusão da variável. 0 oposto aplica-se aos inputs (insumos).

D essa forma, novos indicadores da eficiência DEA são obtidos seqüencialmente pela inclusão de novas variáveis no conjunto de variáveis relevantes.

0 procedimento tem continuidade até que se obtenha um indicador DEA que não se altere com a inclusão de novas variáveis. Isto é, quando a correlação desse indicador com todos os produtos for positiva, e com todos os insumos for negativa. "Apesar de não haver evidências desse raciocínio no texto original de Normam e Stoker, diversos trabalhos referenciam-se ao procedimento (KASSAI, 2000)".

Assim, a maior correlação entre insumos e produtos ocorreu entre as variáveis Requer e D espach $(0,9316)$. Esse foi, portanto, o par inicial input-output do modelo. As demais variáveis de output 
(produtos) foram inseridas no modelo conforme procedimento anteriormente descrito. ICT U, IPD U e IC A apresentam forte relacionamento entre si, o que indica que são redundantes, explicam o mesmo fenômeno. O ptamos por incluir no modelo a variável ICTU, cujo conceito permite aos gestores interpretações mais elaboradas da produtividade na unidade e assume maior peso na avaliação por pontos, adotada pela instituição. Entraram também no modelo, sucessivamente, as variáveis $\mathrm{N}^{\circ}$ Servidores, ID T, TMC, IMA. Dessa forma, obtivemos os insumos Requer e $\mathrm{N}^{\circ}$ Servidores. As demais variáveis foram inseridas no modelo como produtos.

\section{Análise dos resultados}

As D MU: APS - Aracati, APS - Boa Viagem, APS - Canindé, APS - Maranguape, APS - Pacatuba, APS - Q uixeramobim, APS - Redenção - Ce e APS Russas atingiram a fronteira de eficiência. São consideradas, portanto, unidades de referência e definem afronteira de eficiência do conjunto observado. A tabela 1, abaixo,

\section{Tabela 1: Classificação das APS-GEXFOR}

\begin{tabular}{|c|c|c|}
\hline Rank & DMU & Score \\
\hline 1 & Aps Russas & 1 \\
\hline 1 & Aps Aracati & 1 \\
\hline 1 & Aps Redencao-Ce & 1 \\
\hline 1 & Aps Boa Viagem & 1 \\
\hline 1 & Aps Caninde & 1 \\
\hline 1 & Aps Quixcramobim & 1 \\
\hline 1 & Aps Pacatuba & 1 \\
\hline 1 & Aps Maranguape & 1 \\
\hline 9 & Aps Fort-Messejana & 0.96277 \\
\hline 10 & Aps Baturite & 0,955518 \\
\hline 11 & Aps Fort-Parquelandia & 0.853095 \\
\hline 12 & Aps Fort-Aldeota & 0,802968 \\
\hline 13 & Aps Fort-Jacarecanga & 0,792865 \\
\hline 14 & Aps Fort-Centro-Oest & 0,775455 \\
\hline 15 & Aps Fortaleza-Sul & 0,757831 \\
\hline 16 & Aps Quixada & 0,718964 \\
\hline 17 & Aps Pacajus & 0,71378 \\
\hline 18 & Aps Fort-Jangada & 0,691769 \\
\hline 19 & Aps Fort-Parangaba & 0,60125 \\
\hline 20 & Aps Catucaia & 0,595798 \\
\hline 21 & Aps Cascavel-Ce & 0,592883 \\
\hline 22 & Aps Maracanau & 0,558692 \\
\hline
\end{tabular}


mostra a classificação das APS-GEXFOR, segundo os escores obtidos.

O s demais resultados disponibilizados pelo DEA serão discutidos a seguir:

A tabela 2, abaixo, identifica as origens e quantidades de ineficiência em cada APS-GEX FO R. Isto é, mostra os excessos em cada input e a escassez em cada output que tornaram a DMU relativamente ineficiente. Por conseguinte as D MU eficientes têm as colunas ex cessos e escassez iguais a zero.

É importante observar que o método não encontrou nenhuma unidade com excesso na variável Requer. Embora tenha encontrado 8 unidades com excesso na variável $\mathrm{N}$ S ervidores, todas em Fortaleza. A pesar disso, observa-se os pequenos valores nas colunas do IMA r e TM Cr corroborados pelaescassez apresentadana coluna davariável ICTU das unidades ineficientes. Isso indica que as unidades não estão devidamente capacitadas para dar resolver processos e que apresentam baixa produtividade.

O Software DEA-Solver@ disponibiliza ainda as projeções de cada D MU ineficiente na fronteira de eficiência segundo cada variável estudada. Estabelecendo metas e relacionando o potencial de otimização de cada variável da unidade em relação às melhores práticas observadas. Os valores precedidos de sinal negativo representam o excesso que deve ser retirado para tornar a APS eficiente; os valores positivos representam a escassez a ser suprida; e a coluna \% representa 0 percentual que estes valores têm em relação aos seus resultados.

\section{Tabela 2: Excesso e Escassez que tornam a unidade ineficiente no modelo DEA-CCR}

\begin{tabular}{|c|c|c|c|c|c|c|c|c|c|}
\hline \multirow[b]{2}{*}{$\mathrm{N}^{-}$} & \multirow[b]{2}{*}{ DME } & \multirow[b]{2}{*}{ Sorke } & \multicolumn{2}{|c|}{ Fatemin } & \multicolumn{5}{|c|}{ Eivasier } \\
\hline & & & $\begin{array}{c}\text { Recqui } \\
\text { (1) }\end{array}$ & $\begin{array}{c}x^{2} \operatorname{ser} t \\
S-2 \theta\end{array}$ & $\begin{array}{l}\text { Dequach } \\
5+(1)\end{array}$ & $\begin{array}{l}\text { KCnV } \\
s+B z\end{array}$ & $\begin{array}{l}\text { Wr } \\
5+(T)\end{array}$ & $\begin{array}{l}\text { Daing } \\
s+(-4)\end{array}$ & $\begin{array}{l}\text { rater } \\
s+(0)\end{array}$ \\
\hline & Now Ansent & 1 & 0 & 0 & 0 & $\theta$ & 0 & 0 & \\
\hline & Hanern' & 10059918 & a & 11 & (1) & $36 f+160$ & thases & " & 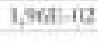 \\
\hline & Aps the Viques & 1 & 6 & $\theta$ & 0 & $\theta$ & 0 & $\theta$ & \\
\hline & Mosinas & 1 & 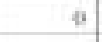 & $\theta$ & a & $\theta$ & 0 & 0 & \\
\hline & $A p=C_{1}=0$ & nesaks & 9 & 11 & i1 & $37 \times 5481$ & 0 & 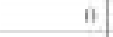 & $1,952-10$ \\
\hline & Ap Cukaia & 10305104 & 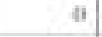 & 11 & 0 & $7 t 5,343 \mathrm{~s}$ & 240,1549 & 144735 & 1 \\
\hline & Ago Fintiniboes & Mvageta & 9 & 65,54k15 & i) & 309,5718 & 34,34542 & 213112 & \\
\hline & 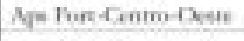 & 077455 & 0 & 200466 & 0 & 175.1314 & 71,45101 & MST: IQ & 35619 \\
\hline & $A$ A Furt Jacaluranye & nTuzsis & 0 & 14221577 & 0 & anzorat & 37,5182 & 2,Jutice & 9,45:-14 \\
\hline ii & Ape firm-jangata & 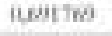 & 8 & 24,73411 & 0) & $127,4 \times 2$ & tirisw4h & $3271-12$ & \\
\hline 11 & As Fun Mlowcinn & 006277 & 9 & 13,90634 & 0 & 23 ients & 98,70128 & 3,50 12 & 4.24E-10 \\
\hline 12 & Ny= Tort-Mraspula & Mat2s & 0 & 108064 & 0 & 3559073 & 1253450 & Mat: 10 & ousich \\
\hline 19 & Aqu thirn Mingratantia & 145ssons & a & 27,95544 & i) & $179+133$ & 42,70349 & Miesent & $2, \sin 10$ \\
\hline 14 & Apo Furndrm Sul & 20757631 & 0 & 4784603 & 0 & $x 50,1407$ & 1249027 & $1,68 \mathrm{E}, 02$ & \\
\hline 15 & Apo Mlarscinan & 0556602 & 0 & 0 & 0 & 171.3693 & 2636008 & 4.CAE 10 & \\
\hline 16 & $\mathrm{Agn}$ Merolgate & & a & 11 & 11 & n & 0 & " & 11 \\
\hline 17 & Aps Prapien & сатын & 0 & 0 & 0 & 17,15ass & 12,27456 & sentera & A.906- 63 \\
\hline 18 & Apo Hacanba & 1 & 0 & $\theta$ & 0 & 0 & 0 & 0 & $\theta$ \\
\hline 19 & Ag= Quesuals & 1871356-4 & 0 & 0 & 0 & 25kelas & $13 \mathrm{x}, \mathrm{ark}$ & \$NQE 18 & 11 \\
\hline 31 & 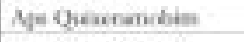 & 1 & $\theta$ & 0 & 0 & 0 & 0 & 0 & \\
\hline 21 & $A p=$ Rodincas - $C_{4}$ & 1 & 4 & $\theta$ & 0 & $\theta$ & 6 & $\theta$ & 11 \\
\hline 22 & Apo Kisus & 1 & 6. & 6 & i) & 0 & a. & i. & 1 \\
\hline
\end{tabular}


Portanto, as D MU eficientes não apresentam diferenças. D eve-se sempre lembrar que a eficiência DEA é relativa ao conjunto das DMU observadas. Encontram-se nesta situação: APS-A racati (tabela 3), APS-BoaViagem, APS-Canindé, APS-Maranguape, APS-Pacatuba, APSQ uixeramobim, APS-Redenção e APSRussas, que têm interpretação semelhante.

A seguir, será analisada cada uma das unidades pela ordem decrescente que aparecem no rank de soores de eficiência do conjunto das 22 D MU estudadas.

A tabela 4 apresenta as projeções da APS-Messejana na fronteira de eficiência, segundo cada variável estudada. Neste artigo, foram omitidas as demais tabelas de projeções, sem prejuízo de informações, uma vez que aquelas decorrem das informações disponibilizadas na tabela 2. A análise dessas informações possibilita que as APS ineficientes definam metas a serem alcançadas para atingir a eficiência relativamente ao conjunto observado.

A APS-Messejana apresenta baixo percentual necessário para a variável $D$ espach atingir a fronteira de eficiência, comparado ao elevado percentual de diferença das variáveis IM A r e T M Cr, o que aponta para a necessidade de melhorar o gerenciamento dos processos na unidade e a capacidade resolutiva.

A APS-Baturité apresenta baixo percentual de diferença para D espach eIM A r em contraste com os altos percentuais de diferença nas variáveis ICT U e ID T, o que

\section{Tabela 3: Projeção - APS - Aracati segundo cada variável estudada}

\begin{tabular}{|c|c|c|c|c|c|}
\hline$N^{\prime \prime}$ & $\begin{array}{l}D M U \\
1 / C\end{array}$ & $\begin{array}{l}\text { 1/Score } \\
\text { Data }\end{array}$ & Projeção & Diferenca & $\%$ \\
\hline \multirow[t]{8}{*}{1} & Aps Aracati & 1 & & & \\
\hline & Estoq & 235 & 235 & 0 & $0,00 \%$ \\
\hline & No.Serv & 15 & 15 & 0 & $0,00^{\mathrm{n}} / \mathrm{s}$ \\
\hline & Despach & 239 & 239 & 0 & $0,00 \%$ \\
\hline & ICTU & 81,16667 & 81,16667 & 0 & $0.00 \%$ \\
\hline & IDT & 92,91 & 92,91 & 0 & $0,00^{1 /} /$ \\
\hline & IMAT & 0,0233 & 0,0233 & 0 & $0,00 \%$ \\
\hline & TMCr & 0,1111 & 0,1111 & 0 & $0,00^{\circ} / \mathrm{e}$ \\
\hline
\end{tabular}

Tabela 4: Projeção - APS Fort-Messejana segundo cada variável estudada

\begin{tabular}{|c|c|c|c|c|}
\hline $\begin{array}{l}\text { DMUU } \\
1 / 0\end{array}$ & $\begin{array}{l}1 / \text { Score } \\
\text { Data }\end{array}$ & Projeçĩo & Diferença & $\%$ \\
\hline Aps Fort-Messejana & 1.03867 & & & \\
\hline Fistoq & 511 & 511 & 0 & $0,00 \%$ \\
\hline No.Sery & 30 & 16,19366 & $-13,8063$ & $-46,02 \%$ \\
\hline Despach & 563 & 584,7711 & 21.77113 & $3.87 \%$ \\
\hline ICTU & 69.9083 .33 & 292,6854 & 222,7771 & $318,67 \%$ \\
\hline IDT & 73.96 & 175,5213 & 101,5613 & $137,32 \%$ \\
\hline IMAr & 0,0116 & $5,00 \mathrm{E}-02$ & $3,84 \mathrm{E}-02$ & $331,21 \%$ \\
\hline TMCr & 0,0345 & $7,83 \mathrm{E}-02$ & 4,38E-02 & $126,87 \%$ \\
\hline
\end{tabular}


pode indicar que um número reduzido de servidores está apto a despachar processos de maior grau de complexidade, o que contribui com reduzido $\mathrm{TMCr}$.

A APS-Parquelândia revela alto percentual de diferença a corrigir navariável $\mathrm{N}$ ' Servidores, comparado ao relativamente baixo percentual da variável $D$ espach e elevados percentuais para ICT U, IMA r e TMCr; o que indica baixa capacidade resolutiva e dificuldade em atender processo de maior grau de complexidade, além de apontar para a necessidade de melhoria no gerenciamento do estoque.

A APS-Aldeota, 12a no ranking, considerada pelos servidores como uma agência privilegiada por ter sido beneficiada em sucessivos programas institucionais de melhoria do atendimento aos usuários, surpreende por todos os percentuais apresentados. Com alto percentual deIM A r e ID T a atingir, embora numericamente próximo do projetado, aparece defasada em relação ao conjunto observado. Alterando a variável $\mathrm{N}^{\circ}$ Servidores da APSAldeota para 19 e fazendo nova simulação, encontramos que a unidade, embora não tenha excessos a corrigir, ainda se mantém na mesma posição no ranking, devido aos resultados obtidos pelas APS-A racati, APSRedenção e APS-Boa Viagem. As duas primeiras já figuravam como referência desta unidade no modelo anterior.

A APS-Jacarecanga apresenta altos percentuais para ID T e IM A r, o que indica dificuldades a vencer quanto à capacitação de servidores. Além disso, apresenta gerenciamento inadequado do estoque, considerando o percentual de $\mathrm{TMCr}$ em relação ao IMA r.

A APS-Fort-Centro-O este mostra dificuldades no gerenciamento do estoque, além de baixa capacidade resolutiva e produtiva. Apresenta também altos percentuais a atingir pelas variáveis ID T, TM Cr e IM A r , cujos reflexos são perceptíveis pelos usuários (prejuízo social), e pela Instituição (prejuízo financeiro).

A APS-Fortaleza-Sul, com altos percentuais de ICTU e ID T a atingir, demonstra capacidade resolutiva aquém do seu porte. Além disso, IM A r e TM Cr na avaliação relativa também deixam a desejar. Isso significa que outras unidades, possivelmente as APSAracati e APS-Redenção suas unidades de referência, obtiveram melhorrelação insumoproduto. Procedeu-se à nova simulação, alterando o quantitativo de servidores para 25. As constatações foram equivalentesàs que se obteve na segunda simulação da APSAldeota. Numa terceira simulação, mantendo-se a variável original $\mathrm{N}^{\circ}$ Servidores (73) e aumentando a variável D espach para 730 (mesmo valor davariável Requer), aAPS salta da $15^{a}$ para a $11^{a}$ posição no ranking, diminuem os percentuais de D espach, ID T eTM Cr a, incrementar nos produtos, mas aumenta 0 excesso de servidores. Isso sugere que, efetivamente, um número reduzido de servidores atua nas atividades-fim.

A APS-Q uixadá expõe capacidade resolutiva inferior a seu porte. O s percentuais de diferença a atingir muito próximos entre IMA r e TMCr apontam para baixa capacidade resolutiva.

A APS-Pacajus apresenta percentuais a atingir relativamente próximos para as variáveis D espach, ICT U , ID T , IM A r eTM Cr - o que pode indicar uma certa equiparação na qualificação dos servidores - porém apontam para a necessidade de aumentar a produtividade. $\mathrm{O}$ percentual IM A r eTM C r a alcançar podem revelar a necessidade de melhorar o gerenciamento do estoque.

A APS-Jangada mostra altos percentuais a atingir em ID T e IMA r. Um baixíssimo resultado obtido pela variável ICTU demonstra que a unidade enfrenta 
problemas na capacitação resolutiva e na produtividade. Pode indicar também que um número reduzido de servidores atua nas atividades-fim.

A APS-Fort-Parangaba apresenta altos percentuais a alcançar pelas variáveis ID T, IMA r e TMCr, apontando baixa capacidade resolutiva e baixa produtividade da unidade, além das questões de gerenciamento do estoque. Os altos percentuais de diferença a atingir pelas variáveis ICTU, ID T, IMA r e TMCr reforçam a indicação de baixa capacidade resolutiva e produtiva, possivelmente, agravando e dificultando as questões de gerenciamento do estoque. Esta unidade acumula prejuízos sociais (estoque acima de 30 dias) e prejuízo financeiro (estoque acima de 45dias).

A APS Caucaia evidencia um quadro difícil. Os altos percentuais de diferença a atingir pelas variáveis ICTU, ID T, IM A r e TMCr apontam capacidade resolutiva e produtiva muito inferiores ao porte da unidade, 0 que se agrava pela ineficiência no gerenciamento do estoque. Já a APS Cascavel apresenta o mesmo percentual a atingir para ID T, IM A r e D espach. As diferenças maiores a incrementar nos produtos são relativas às variáveis ICT U e T M Cr. Isso denota capacidade resolutiva e produtividade aquém em $103,85 \%$ e $148,12 \%$, respectivamente, das melhores práticas observadas no conjunto. A APS-Maracanau, além da baixa capacidade resolutiva e produtiva, apresenta alto índice de IMA r a alcançar, o que aponta, principalmente, para a ineficiência no gerenciamento dos processos, acarretando prejuízo financeiro à instituição, bem como prejuízo social.

\section{Conclusões}

A avaliação de eficiência das APSGEXFOR, segundo a metodologia DEA, permitiu identificar as melhores práticas, mensurar a participação de cada variável envolvida na avaliação da unidade e projetar as unidades ineficientes na fronteira de eficiência, estabelecendo metas a partir da identificação de percentuais de acréscimos e/ ou decréscimos a serem aplicados nos inputs e/ ou outputs para torná-las eficientes.

Nesse sentido cabe ressaltar a eficiência da APS-Redenção, referenciada 13 vezes
"A avaliação de eficiência nas APS, utilizando a metodologia DEA, poderá contribuir com o processo, já em curso na instituição, de busca por uma gestão pública mais ética, participativa, descentralizada, onientada por resultados."

no conjunto observado. Com oito servidores efetivamenteno quadro (um servidor cedido), sendo dois agentes administrativos, dois técnicos previdenciários, um agente de portaria e três peritos médicos, atenderam, em novembro de 2006, 97,55\% da demanda; e a unidade tem o IMA e o TMC em torno de 35 e 23 dias, respectivamente. A APS-Russas, a segunda maior unidade no interior, atendeu $97,75 \%$ 
da demanda com o IMA e o TMC em torno de 54 e 15 dias, respectivamente.

Ressalta-se ainda que a metodologia DEA analisa a eficiência de unidades relativamente ao conjunto observado. Significa dizer que, incluindo-se ou excluindo-se unidades nesse conjunto, poderíamos obter scores diferentes. Uma unidade eficiente em um conjunto poderia não ser eficiente em outro. Reside também nesse conceito a força da metodologia, que possibilita avaliar o grau de eficiência relativa de unidades produtivas que realizam uma mesma atividade quanto à utilização dos seus recursos. Neste trabalho, estudamos as 22 APS da Gerência Executiva Fortaleza - CE.

Nenhuma unidade da capital atingiu a eficiência relativa, não obstante o reconhecido perfil dos servidores em algumas unidades e a proximidade à Divisão de Benefício, aos Serviços de Benefício e ao Serviço de Atendimento. Em simulações subseqüentes realizadas para as APSFortaleza Aldeota e APS-Fortaleza-Sul, onde se reduziu o quantitativo de servidores aos números projetados pela metodologia DEA, não se observou mudança significativa nos sores de eficiência destas unidades, que se mantiveram com as mesmas classificações no ranking. E ssas classificações foram, no entanto, significativamente alteradas quando mantido 0 último quantitativo de servidores eaumentando os [outputs] D espach e ID T.

A utilização da metodologia DEA, instrumento de apoio à decisão de natureza multicritério, permitiu definir a eficiência de cada APS de forma individualizada, considerando a atuação das demais unidades em estudo. A metodologia também propôs melhorias com o estabelecimento de metas de atuação, possibilitou ainda 0 estudo dos fatores que mais contribuíram para a obtenção da eficiência relativa. Diferentemente dos sistemas de atribuição de pontos, mais de uma unidade pôde ser classificada como eficiente, compondo a fronteira de eficiência relativa e servindo como referência às demais unidades. Isso permite identificação das melhores práticas, importante aliada no processo de gestão participativa e contribui para a aprendizagem organizacional.

A identificação do perfil dos servidores das APS-GEXFOR poderia colaborar significativamente para aumentar do poder discricionário do modelo, permitindo identificar as reais necessidades de treinamento, o quantitativo de servidores que efetivamente desenvolvem as atividadesfim e a lotação eficientemente adequada de servidores nas unidades.

O software DEA-Solver@ utilizado para implementar a aplicação mostrou-se extremamente amigável para o usuário e de fácil utilização.

A avaliação de eficiência nas Agências da Previdência Social, utilizando a metodologia DEA, poderá contribuir com o processo, já em curso na instituição, de busca por uma gestão pública mais ética, participativa, descentralizada, orientada por resultados. A inserção nesse contexto de ferramentas científicas e tecnológicas, como a Pesquisa Operacional e os métodos de Apoio à Decisão Multicritérios metodologia DEA, oferece instrumentos de avaliação continuada de gestão, possibilitando a condução da implementação de ações para assegurar a melhoria da gestão dos serviços públicos prestados ao cidadão.

A utilização da metodologia DEA para avaliação de eficiência em unidades de atendimento pode atender à demanda dos administradores do setor público que, segundo Machado(2005), "necessitam de 
um conjunto de informações gerenciais para cumprir, com eficiência, eficácia e efetividade, as políticas públicas". Corrobora também com Trosa(2001), que argumenta que "a busca do desempenho pode simultaneamente definir as margens de manobra financeira ou de eficiência e atender à preocupação dos servidores em ter um trabalho mais valorizado", uma vez que permite a avaliação continuada das ações implementadas, o que possibilita, também, a ampliação da visão do processo. Isso poderá contribuir significativamente com a modernização da gestão pública pela implementação de medidas que assegurem a eficiência, redução de custos operacionais e melhoria na qualidade do atendimento aos cidadãos.

(Artigo recebido em setembro de 2007. Versão final em junho de 2008).

\section{Referências bibliográficas}

Brasil. Ministério do Planejamento. Plano PluRianual (PPA) 2004-2007, disponível na URL: http:/ / www.planobrasil.gov.br

Cooper, William W., Seiford, Laurence, Tone, Kaoru - Data Envelopment Analysis A comprehensive Text with Models Applications, References and DEA - Solver Software, Kluwer Academic Publishers, Second Printing 2000.

França, Álvaro Sólon de, Previdência Social e a Economia dos Municípios, DF, 5a ed. ANFIP, 2004.

Goldbarg, Marco César; Luna, Henrique Pacca L., O timização Combinatória e Programação Linear: modelos e algoritmos, Rio de Janeiro, Ed. Elsevier, 2000.

Gomes, Luiz Flávio Autran Monteiro, Gomes, Carlos Francisco Simões e Almeida, Adiel Teixeira. Tomada de Decisão Gerencial - Enfoque Multicritério. $2^{\circ}$ edição - Ed. Atlas. São Paulo. 2006.

KASSAI, Silvia. Utilização da Análise por Envoltória de Dados (DEA) na Análise de D emonstrações Contábeis. 2002. Tese (D outorado) - Faculdade de Economia, Administração e Contabilidade e Atuária da Universidade de São Paulo.

Lins, Marcos P. E. e MeZA, Lídia A. Análise Envoltória de Dados e perspectivas de interação no ambiente de apoio à decisão - Ed. COPPE-UFRJ, 2000.

MACHAdo, Nelson. Sistema de Informação de Custo - diretrizes para integração ao orçamento público e à contabilidade governamental. Brasília. ENAP, 2005.

MezA, Lídia Angulo. D ata Envelopment Analysis (DEA) na determinação da eficiência dos programas de pós-graduação da CO PPE/ UFRJ. 1998. Dissertação (Mestrado) Programa de Pós-Graduação em Engenharia da Universidade Federal do Rio de Janeiro. Rio de Janeiro: COPPE/ UFRJ.

Neiderauer, Carlos Alberto Pittaluga. Avaliação dos bolsistas de Produtividade em Pesquisa de Engenharia de Produção utilizando DATA Envelopment Analysis. 1998. Dissertação (Mestrado) - Programa de Pós-Graduação em Engenharia de Produção da Universidade Federal de Santa Catarina, Florianópolis: UFSC. 
PaIva, Francisco Canindé de. Eficiência produtiva de programas de ensino de pósgraduação em engenharias: uma aplicação do método Análise Envoltória de Dados DEA. 2000. Dissertação (Mestrado). Programa de Pós-Graduação em Engenharia de Produção da Universidade Federal de Santa Catarina. Florianópolis-UFSC.

Reinaldo, Reinaldo Richard Portela - Avaliando a Eficiência em Unidades de Ensino Fundamental de Fortaleza-CE, D issertação (mestrado) - Programa de Pós-G raduação em Engenharia de Produção da Universidade Federal de Santa Catarina, 2002.

Silva, Antonio Carlos Magalhães da. Análise da eficiência das Instituições financeiras brasileiras, segundo a metodologia do Data Envelopment Analysis (DEA). 2000. Dissertação (Mestrado) - Instituto de Pós-G raduação em Administração, da Universidade Federal do Rio de Janeiro. Rio de Janeiro: CO PPEAD/ UFRJ.

Trosa, Sylvie - Gestão Pública por Resultados: quando o Estado se compromete, tradução: Maria Luiza de Carvalho, Rio de Janeiro: Revan; Brasilia, DF: ENAP, 2001. 


\section{Resumo - Resumen - Abstract}

\section{Gestão Pública e Pesquisa Operacional: avaliação de desempenho em Agências da Previdência Social}

A delina Cristina A . Chaves e A . Clecio F. T homaz

O presente trabalho utiliza a metodologia DEA (D ata E nvelopment A nalysis ), para avaliar a eficiência das 22 A gências da Previdência Social - G erência Executiva Fortaleza (APS-GEX FO R). DEA utiliza programação linear cujo núcleo das estruturas analíticas é formado a partir do modelo original CCR (Charnes, Cooper, e Rhodes). A plicada às D MU (D ecision M aking U nits), define uma Fronteira de Eficiência identificando Unidades Eficientes e Ineficientes. Foi utilizado o modelo D EA-CCR implementado pelo sottware DEA Solver@ . A Previdência Social (INSS) dispõe de indicadores de desempenho. Algumas variáveis utilizadas no modelo implementado derivam desses indicadores, outras informações foram disponibilizadas pelos sistemas de informação da instituição. A avaliação de eficiência D EA das APS-GEXFOR permitiu identificar as melhores práticas, mensurar a participação de cada variável envolvida na avaliação da unidade e projetar as unidades ineficientes na fronteira de eficiência, identificando metas a serem atingidas para torná-las eficientes no conjunto observado.

Palavras-chave: DEA (Análise Envoltónia deD ados), Eficiência, G estão Pública por resultados, INSS-Agências da Previdência Social.

\section{Gestión Pública e Investigación Operacional: evaluación de desempeño en Agencias de la Previdencia Social}

A delina C ristina A . C haves y A . Clecio F. Thomaz

El presente trabajo utiliza la metodología DEA (D ata E nvelopment A nalysis), para evaluar la eficiencia delas 22 Agencias dela Previdencia Social - G erencia Ejecutiva Fortaleza(APS-GEXFOR). DEA utiliza programación linear cuyo núcleo son las estructuras analíticas y el modelo original CCR (Charnes, Cooper, e Rhodes). A plicada a las D MU (D ecision M ak ing U nits), define una Frontera de Eficiencia identificando Unidades Eficientes e Ineficientes. Fue utilizado el modelo D EA-CCR implementado por el software D EA Solver@ . La Previdencia Social (INSS) dispone de indicadores de desempeño. Algunas variables utilizadas en el modelo implementado derivan de estos indicadores. O tras de las informaciones también fueron disponibilizadas por el sistema de información de la Institución. La evaluación de eficiencia DEA de las APS-G EXFO R, permitió identificar las mejores prácticas, medir la participación de cada variable envuelta en la evaluación de la unidad y proyectar las unidades ineficientes en la frontera de eficiencia, identificando metas a ser alcanzadas para tomarlas eficiente en el conjunto observado.

Palabras-clave: DEA (Análisis Envoltorio deD atos), Eficiencia, G estión Pública por resultados, INSS- Agencias dela Previdencia Social.

\section{Public Management and 0 perational Research: performance evaluation of Agencies of Social Security}

A delina C ristina A . Chaves and A . Clecio F. Thomaz

This work utilizes DEA (D ata Envelopment A nalysis) methodology to evaluate the efficiency of the 22 Agencies of Social Security in Fortaleza-BRAZIL (APS-G EX FOR). D EA utilizes linear programming whose analytic structures nucleus is formed from the original model CCR (Charnes, Cooper, Rhodes). When applied to D MU (D ecision Making Units), it defined inefficient and efficient 
units, drawing an Efficiency Border. In the application, the model DEA-CCR was used and it implemented by the software DEA-Solver@). The Social Security agency (INSS) has an indicator of performance. Some variables in the model implemented derive from these indicators. O thers derive from information disposed by the Information Systems Corporation. D EA evaluation of efficiency of the APS-G EXFOR permitted: to identify the best practices, to measure the participation of each variable involved in the unit evaluation, and to project the inefficient units in the border of efficiency, identifying goals to be reached in order to become efficient among the units observed.

Key words: DEA(D ata Envelopment A nalysis), Efficiency, Results- $O$ riented Public Management, Agencies of the Social Security - INSS (National Institute of Social Security of the Brazil).

Adelina Cristina A. Chaves

INSS - Gerência Executiva Fortaleza.

Contato: adelinacristina@gmail.com

A. Clecio F. Thomaz

Universidade Estadual do Ceará - LOGIN Laboratório de Otimização e G estão Industrial.

Contato: clecio@larces.uece.br 